\section{Penetrating membranes}

Several models have been proposed to explain how pro- and antiapoptotic family members, despite sharing extensive structural similarity and interacting with 'activating' BH3-only members, differ in function. In PLoS Biology, Billen et al. show that interactions of $\mathrm{Bax}, \mathrm{Bcl}-\mathrm{x}_{\mathrm{L}}$ and Bid, which are proapoptotic, antiapoptotic and activating family members, respectively, occur on membranes to either promote or inhibit loss of membrane integrity. $\mathrm{Bcl}-\mathrm{x}_{\mathrm{L}}$ that binds to Bid undergoes a conformational change, which allows its tethering to membranes. Likewise, after binding Bid, Bax undergoes membrane insertion and can form oligomers with itself to create membrane pores. Bcl- $x_{\mathrm{L}}$, however, cannot undergo this process, fails to form pores and, notably, can 'out-compete' Bax for both Bid binding and recruitment of monomeric Bax molecules. Bcl- $x_{\mathrm{L}}$ and, presumably, other antiapoptotic $\mathrm{Bcl}-2$ members thus act as dominant negative molecules to inhibit proapoptotic pore-forming activity. PLoS Biol. (10 June 2008) doi:10.1371/journal.pbio.0060147

\section{Manipulating vesicle transport}

Although uncommon in mammalian cells, ankyrin repeat homology domain proteins are abundant in bacterial pathogens. In Science, Roy and colleagues show that such a protein in Legionella pneumophila, AnkX, acts as a virulence factor by virtue of its ability to manipulate host cell vesicle trafficking. After being transported into the host cytosol by means of the DotA type IV secretion system, AnkX blocks endoplasmic reticulumto-Golgi vesicle transport. Like the microtubule-depolymerizing agent nocodazole, AnkX also suppresses transport of endocytic vesicles to the perinuclear area. Vacuoles enveloping L. pneumophila mutants lacking AnkX show more fusion with lysosomes; this defect is 'rescued' by treatment with nocodazole. Thus, by competing with the Golgi for recruitment of secretory vesicles needed to remodel bacteria-containing vacuoles, and by blocking microtubule-mediated transport of these vacuoles toward destructive lysosomes, AnkX promotes bacterial virulence.

Science 320, 1651-1654 (2008)

\section{PD-L1 in $\mathrm{T}_{\text {reg }}$ development}

Naive $\mathrm{CD}^{+} \mathrm{T}$ cells exposed to transforming growth factor- $\beta$ (TGF- $\beta$ ) can convert into 'adaptive' Foxp $3^{+}$regulatory T cells ( $\mathrm{T}_{\text {reg }}$ cells) and thereby contribute to peripheral tolerance mechanisms. In the Proceedings of the National Academy of Science, Noelle and colleagues show that immature CD8 $\alpha^{+}$ dendritic cells (DCs) have a greater capacity to induce $\mathrm{aT}_{\text {reg }}$ cells in the presence of TGF- $\beta$ than do other DC subtypes. Unexpectedly, they find that the coinhibitory molecule PD-1 is also required for TGF- $\beta$-induced $\mathrm{aT}_{\text {reg }}$ conversion. The interaction of PD-1 with its ligand PD-L1 but not with PD-L2 promotes Foxp3 expression in $\mathrm{T}_{\text {reg }}$ cells. Blockade of PD-1-PD-L1 in a tumor model delays tumor growth. How the PD-1 pathway intersects with TGF- $\beta$ signaling to induce regulatory cells remains to be determined.

Proc. Natl. Acad. Sci. USA 105, 9931-9336 (2008)

\section{A DEAD-box TANK}

During infection many pathogen-associated molecules stimulate IFN$\beta$ production through a signaling pathway involving TANK-binding kinase 1 (TBK1) and interferon-regulatory factor 3 (IRF3). In $E M B O$ Journal, Soulet et al. find that the X chromosome-encoded DEAD-box helicase DDX3X is a TBK1 substrate in vitro and in vivo that is necessary for IFN- $\beta$ production after infection with Listeria monocytogenes or stimulation with innate signaling molecules such as lipopolysaccharide, poly(I:C) or poly(dA:dT). Decreasing DDX3X expression by RNA-mediated interference results in less production of IFN- $\beta$ but not tumor necrosis factor. Phosphorylation of DDX3X by TBK1 occurs in the DDX3X DEAD domain and is required for recruitment of DDX3X to the Ifnb promoter, where IRF3 is also recruited, to induce IFN- $\beta$ expression. Further work is needed to determine the importance of DDX3X during viral infection.

$D C B$

EMBO J. (26 June 2008) doi: 10.1038/emboj.2008.126

\section{Enabling Ifng transcription}

In addition to $\mathrm{T}$ cell receptor (TCR) signals, cytokine signals that activate the transcription factor T-bet are essential for the differentiation of $T$ helper type $1\left(T_{H} 1\right)$ cells producing interferon- $\gamma$ (IFN- $\gamma$ ). In Immunity, Berg and colleagues identify cytokine signals activating the kinase Jak3 and the transcription factor STAT5 as another requirement for $\mathrm{T}_{\mathrm{H}} 1$ cell development. Jak3- and STAT5-deficient CD4+CD8- thymocytes show impaired TCR-driven IFN- $\gamma$ production in neutral as well as $\mathrm{T}_{\mathrm{H}}$ 1-polarizing conditions. Neutralization of interleukin 2 also suppresses $T_{H} 1$ differentiation. Although expressed in normal amounts, T-bet binds less efficiently to the Ifng promoter in Jak3-deficient CD4 ${ }^{+} \mathrm{CD} 8^{-}$thymocytes; these cells also have less acetylation of histone $\mathrm{H} 3$ at the Ifng promoter. Treatment with a Jak3 inhibitor suppresses $\mathrm{T}_{\mathrm{H}} 1$ differentiation when added during but not after $\mathbf{4 8}$ hours of TCR stimulation, which suggests that interleukin 2-induced Jak3 and STAT5 act before or synergistically with T-bet to facilitate chromatin remodeling at the Ifng locus.

Immunity 28, 763-773 (2008)

\section{RIG-Ing viral RNA}

Infection of the liver with hepatitis $\mathrm{C}$ virus (HCV) activates IRF3 to induce expression of IFN- $\alpha / \beta$ and interferon-stimulated genes that limit infection. In Nature, Gale and colleagues identify the polyuridine motif of the HCV RNA genome 3' untranslated region as a substrate of the RNA helicase RIG-I that activates interferon signaling pathways that are independent of the helicase MDA5 and the adaptors MyD88 and TRIF. The polyuridine motif of HCV induces stable interaction with RIG-I that depends on the $5^{\prime}$ terminal triphosphate end of the RNA. This motif is both necessary and sufficient to trigger hepatic innate immune responses in vivo, and conserved 5' terminal triphosphate-containing polyuridine motifs in rabies virus, Ebola virus and measles virus similarly trigger IFN- $\alpha / \beta$ production. These data demonstrate that the polyuridine motifs found in many RNA viruses are

$D C B$ critical features for RIG-I recognition.

Nature (11 June 2008) doi:10.1038/nature07106

$L A D$

(Nature (11 June 2008) doi:10.1038/nature07106 